\title{
Mobile Access to Personal Digital Photograph Archives
}

\author{
Cathal Gurrin ${ }^{1}$, Gareth J.F. Jones ${ }^{1}$, Hyowon Lee ${ }^{1}$, Neil O'Hare ${ }^{1}$, Alan F. Smeaton ${ }^{2}$, Noel Murphy ${ }^{1}$ \\ ${ }^{1}$ Centre for Digital Video Processing, ${ }^{2}$ Adaptive Information Cluster \\ Dublin City University \\ Dublin, Ireland \\ cgurrin@computing.dcu.ie
}

\begin{abstract}
Handheld computing devices are becoming highly connected devices with high capacity storage. This has resulted in their being able to support storage of, and access to, personal photo archives. However the only means for mobile device users to browse such archives is typically a simple one-by-one scroll through image thumbnails in the order that they were taken, or by manually organising them based on folders. In this paper we describe a system for context-based browsing of personal digital photo archives. Photos are labeled with the GPS location and time they are taken and this is used to derive other context-based metadata such as weather conditions and daylight conditions. We present our prototype system for mobile digital photo retrieval, and an experimental evaluation illustrating the utility of location information for effective personal photo retrieval.
\end{abstract}

\section{Categories and Subject Descriptors}

H.5.1 [Information Interfaces and Presentation]: Multimedia Information Systems - evaluation/methodology.

\section{General Terms}

Human Factors.

\section{Keywords}

Personal Digital Photo Archive, GPS Location, Mobile Access.

\section{INTRODUCTION}

Recent years have seen a revolution in photography with a move away from analog film towards digital technologies, resulting in many users accumulating very large numbers of personal digital photographs. The standard scenario is that these are uploaded from a camera to be stored, accessed and printed using personal computers. However, the increasing storage capacity of handheld devices, such as the iPod Photo, and the cameras themselves means that mobile users are increasingly now actually carrying significant photo archives with them in their pockets. In addition, many web services exist that offer photos storage and basic organisation services for a user's digital photos.

Developments in wireless networking support users downloading photos from their personal archives (and elsewhere) to handheld devices whilst the user is mobile. This provides a great opportunity for users to share photos with others while on the move, but a significant issue is how does a mobile device support searching through a personal archive which may comprise hundreds or thousands of photos?

In this paper we describe how we use contextual data, e.g. time and location of image capture, to manage a personal digital photo archive. We illustrate how this is particularly valuable for search on mobile devices where data entry is restricted. The time and date of capture are easily accessible from the camera, and this can be augmented with location information using coincident GPS location data. Using context in this way has the advantage that photo indexing is entirely automatic, thereby removing the need for the user to manually annotate and index the photos.

The mobile system described in this paper gives a user ubiquitous access to the contents of their photo archive. We also show experimentally that using context annotation in this way can vastly improve retrieval speed, reducing the amount of user interaction required to locate relevant photos by nearly $50 \%$.

\section{EXISTING MANAGEMENT TOOLS FOR DIGITAL PHOTOGRAPH ARCHIVES}

In this section we briefly review currently available tools for management of digital photo archives.

\subsection{Conventional Desktop Access}

There are many commercial photo management tools available and most of these have been developed for desktop computing environments. In mid-2004 we evaluated the functionality of twenty popular Windows-based tools ${ }^{1}$ and concluded that all perform the same basic management function, managing photos using a photo album or folder metaphor and displaying thumbnails, and some providing calendar views to support organisation. We also concluded that there exists a strong reliance on users to manually annotate or categorise images, which is later used to support retrieval.

In addition to commercial products, there has also been an increasing amount of research in the area of personal photo collection management. Some systems attempt to leverage the techniques of Content-Based Image Retrieval to enhance the annotation process [12]. The MiAlbum system [15] uses a semi-

\footnotetext{
1 Including popular tools such as Adobe Photoshop Album, Picasa and Paint Shop Pro Album.
} 
automatic approach to image annotation. In addition, it is possible to exploit the 'bursty' patterns of photo capture to detect bursts of capture activity corresponding to an event such as a birthday party [1]. More recently, location metadata for indexing photo collections has been explored, with the WWMX [14] allowing navigation of photo collections using a map-based interface.

\subsection{Mobile Access}

Mobile platforms impose a number of acknowledged design constraints including: small display size, awkward methods of data input and distractive environments [5, 11, 8]. A photo management tool for mobile platforms must take account of these factors, therefore it is not advised to show a large number of photos on screen at any one time, or to rely on very extensive browse/search functionality. Notwithstanding these limitations, some photo browsing software has been developed specifically for mobile devices. These applications include the HP Image Zone software for the iPAQ where a user can edit photos and display them in a slideshow right on their iPAQ, but can do little more. Also, there has been some previous research in the area of photo management on mobile devices, e.g. [3] which use calendar-based timelines and event-based summarisation to allow for efficient time-based browsing of photo collections.

There are also several web based photo management solutions where users are granted access to a small amount of web space to upload photos and browse from any location. A small number of online digital photo storage services (e.g. Snapfish) support mobile access to a personal photo archive, but require the user to scroll through large numbers of photo thumbnails or search using manually annotated metadata.

\section{CONTEXT AWARE LABELING OF DIGITAL PHOTOGRAPH ARCHIVES}

The basis for our digital photo management application is the automated labeling of image context, in particular using the time and location of photo capture and in addition, using this to derive additional context information. These dimensions of context can be used independently or in combination to support photo retrieval. In this section we outline how we accomplish this.

The key benefits of labeling digital photos with their location are that it enables us to support a number of access methodologies: search by actual location (county, town/city, even street), and search by proximity to a location or to other photos. By using such information the browsing space (number of photos that a user has to browse through) when seeking a particular photo can be drastically reduced.

By integrating location with time, additional metadata can be generated which can further automatically annotate the digital photo. Given an archive of digital photos with location and time metadata each photo can be automatically tagged with additional annotations such as 'daylight status' or weather [7]. A photo taken at $10 \mathrm{am}$ will be daylight in most parts of the world, but this is not always the case, for example, in parts of Scandinavia and similar high latitude locations this time could signify dawn, or even darkness, depending on the time of year. We use standard astronomical algorithms to calculate sunrise/sunset times for any location on any date and using these algorithms we can associate a daylight status (daylight, darkness, dawn or dusk) with each photo and thereby automatically annotate each photo with this information.

Another feature that we use to annotate each photo is weather. There are 10,500 international weather stations dotted all across the globe which constantly log weather data a number of times each day. Given this information, and readily available access to the weather data logs via the WWW, we annotate each photo with the weather data (clear, cloudy, rainy or snowy) from the closest international weather station at the time the photo was taken.

\subsection{Location-Aware Archive Construction}

In order to support our research (including the experiment described below), we collected a large archive of location and time stamped personal photos. While awaiting the arrival to market of consumer-grade digital cameras with location stamping capabilities, we utilise a separate GPS device to record the location at which photos are taken. When uploading photos to our archive, we automatically match photos with their location from a GPS tracklog. In order to map raw GPS co-ordinates to real world locations, we utilise a gazetteer based on the USGS and NGA datasets. This allows us to index each photo at three separate levels; country, city/state and town. Thus far, our archive of location-stamped personal photos consists of more than 8,000 images. These photos have been taken in 16 different countries representing 623 separate locations within these countries. There are 11 users contributing to this archive with an average of 735 images donated by each user.

\subsection{Effectiveness of Location-Aware Photograph Organisation}

Prior research into managing personal digital archives [10] identifies the following three different types of searches applicable to personal collections; (1) searching for all photos associated with a certain event, (2) searching for a specific known photo or (3) searching for all photos sharing some characteristic (e.g. containing a certain person). For this experiment, we restricted our evaluation to users searching for a specific known photo, although our findings would be equally applicable to searching for all photos relating to a certain event.

A key assumption of our work is that integrating context, using location, will better support user search and browsing, both on desktop and mobile devices, than time-based organisation alone. In order to test this assumption we conducted an experiment using a desktop photo archive searching system. This required the use of two versions of our desktop interface to evaluate if and how beneficial location information is to a photo archive:

- Location-aware: a fully functional system using time and location information.

- Conventional: using time information only.

A number of photos for each user were selected (in advance) for use as known item queries and we used seven users for this experiment, with both systems being employed interchangeably. Each user processed different numbers of queries, from 36 to 8 (depending on the size of that user's personal archive). Each query photo was briefly verbally described to the user before searching. If the user failed to recall the photo based on description alone, then he/she was shown the photo. 
For each user we measured the time taken to find each photo and the number of times the query needed to be reformulated (query clicks required). The results for average search time are summarised in Figure 1, where average search time is plotted against collection size for each user. As expected, searches using location are consistently faster than using time alone, but for 4 of the 7 users searches were approximately twice as fast. In all but one case, the location information was shown to improve retrieval effectiveness, with the exception being the user with the smallest collection, where desktop browsing through a small collection of images proved faster than preparing a location-based query.

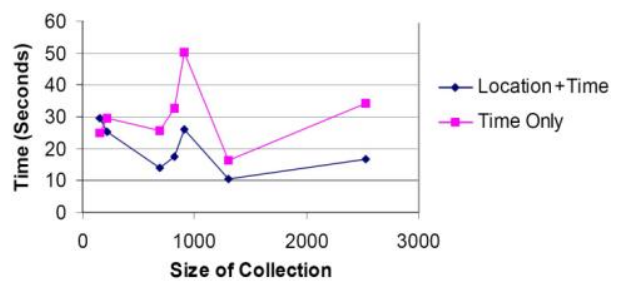

Figure 1. Average Search Time per User

The average time taken to locate a known photo using the location-aware system was 17.9 seconds compared to 31.2 seconds for the time-only system. In terms of query clicks, the average number of query iterations when using the location interface was 2.8 as opposed to 3.7 for time-only. With regard to user satisfaction, we found (by means of a post-experiment questionnaire) that users rated location information an average score of 4.6 ( $\max 5.0)$ compared to 3.0 for the time-only system.

This small experiment clearly illustrates how useful location information is to an archive of digital photos. The result is not unexpected but the magnitude of the difference is surprising. Even with this small experiment we have shown that it is possible to drastically reduce the user interaction and by extension, the cognitive load when querying and browsing location-aware archives. This is a key design consideration for us when supporting mobile access to a personal photo archive, where a user has to overcome the limitations of using a mobile device.

\section{HCI ISSUES FOR MOBILE ACCESS TO DIGITAL PHOTOGRAPH ARCHIVES}

There have been many suggestions on devising interaction paradigms suitable for mobile environments, rather than simply following the conventional direct manipulation interfaces successfully used in desktop platforms [4, 6, 9]. The general consensus is that mobile interfaces often require a different interaction style from that of the GUI desktop interface, and that attempts to replicate all the functionality of the desktop system into a mobile device are a mistake $[13,6,9,5]$. This is something we ourselves have observed when developing a mobile device interface to a video browsing and playback system [2].

We have followed a number of design guidelines that have been suggested in the literature, when developing our own mobile interface to personal photo libraries. These include the following:

- Minimise user input where applicable, provide simple user selections or simple hyperlinking, instead of asking the user to articulate query formulation or use visually demanding browsing that requires careful inspection of the screen;
- Filter out information so that only a small amount of the most important information can be quickly and readily accessed via the mobile device;

- Proactively search and collect potentially useful pieces of information for a user and present these.

In terms of developing any system for a mobile device which is to support searching and retrieval tasks, these guidelines point to providing a small number of interface elements, which require minimal user interaction in order to locate relevant content. Our mobile interface follows these guidelines by supporting the following access methodologies:

- 'My Favorites', the first screen a user sees when accessing their archive using a mobile device (see Figure 2). This is a personalised thumbnail listing of the top ten most popular photos based on a user's history of viewing full-size photos.

- Search functionality, primarily based on location and automatic annotations. The aim is to reduce the level of user interaction required to quickly locate relevant content. In order to maximise screen real-estate available for browsing the photo archives, search options are hidden in a slider panel that slides into view when a user wants to search (see Figure 3) and then disappears afterwards until required again (Figure 2).

- Browsing the collection. Even by supporting the two access methods above, on a mobile device a user may still end up having to spend time scrolling through screens of photos if many were taken at the same place and time. To address this, we present results to the user clustered into events and ordered by date and time.

\subsection{Mobile Interaction}

The mobile interface we present here is twinned with a desktop interface that we used in the experiment described above in that both provide a web-based interface to the same underlying system but interfaces tailored to the individual requirements for each device. The functionality of the desktop interface (not described here) differs greatly from the mobile interface, though also heavily relies on location.

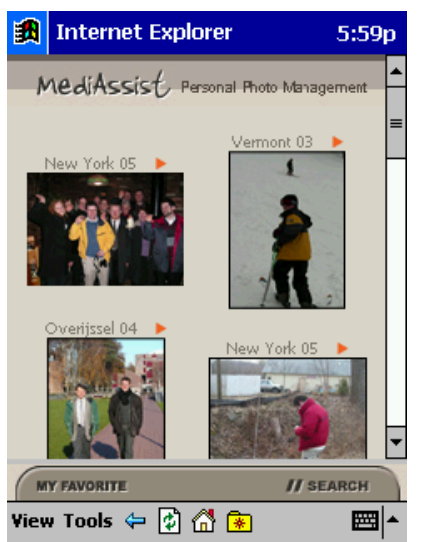

Figure 2. 'My favorite photos' with search frame

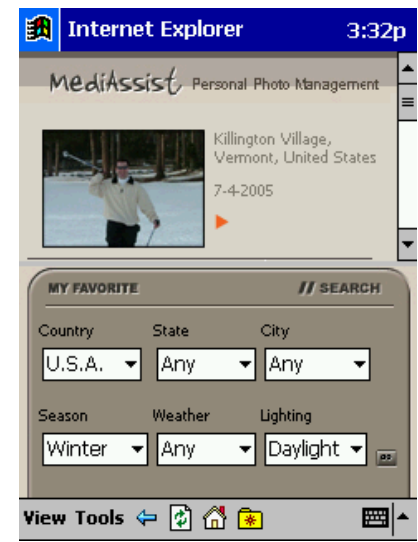

Figure 3. Searching the archive with results as events. 
A user accessing their photo archive is immediately (as stated above) presented with the 'My Favorites' screen, of their most accessed photos, helping to reduce user interaction. If the required photo is not in the favourites, the user engages in a process of search and retrieval, followed by browsing the search results, so in effect it is a two-phase search. The search options are: three level location (country, state/city and town), weather, lighting status and season, as shown in Figure 3. The contents of the location drop-down boxes are personalised to the user's collection to minimise user input. Weather, lighting status and season are included to filter the search results thereby reducing the amount of user browsing required to locate the required photo. Based on a previous user study [7] these were shown to be among the most utilised user features.

Simply presenting a (potentially large) list of photo thumbnails in response to a query is not an ideal interaction scenario for the user of a mobile device. Reducing the size of the thumbnails will display more images on the screen, but at the expense of lower image quality and increased user cognitive load. A more 'mobile friendly' technique is to group photos into events and using a single 'key' thumbnail, represent an event, as shown in Figure 3 (one event displayed). This is done by automatically grouping photos together into logical groups by examining when and where clusters of photos co-occur and choosing a single representative photo to represent the whole cluster. These clusters are then presented to the user, ordered by time/date.

Selecting a thumbnail photo on screen will present the user with a full-screen photo, and it is this detailed viewing of a photo that is used to support the 'My Favorites' access method. This is also the case on the desktop interface, thus allowing the same 'My Favorites' to be used seamlessly on each device. Associated with each thumbnail is also a small orange arrow (see Figures 2, 3). Clicking this arrow will bring the user to a screen showing all photos from that particular photo event, once again organised by date/time. These are presented to the user as a simple listing of images (like in Figure 2), though without the browse event arrow.

\section{CONCLUSIONS \& FUTURE WORK}

In this paper we have presented an interface to an archive of personal photos which is designed for a mobile device. The design considerations have been informed by an experiment which shows how location-aware archives of personal photos reduce user effort in locating desired photos, as well as by the usual design considerations for mobile devices.

We believe that the user interaction presented here is still not complete or optimised. Some form of browsing the archive dynamically could be added by allowing a user to link to spatially similar or graphically similar photos (based on content analysis), or to photos containing the same person or object. This is the subject of our further research. The event detection discussed in section 4.1 is also the subject of ongoing research and will be improved further, along with improved methods of choosing the key image for an event.

\section{REFERENCES}

[1] Cooper, M., Foote, J. and Girgensohn, A. Automatically organizing digital photographs using time and content. In Proc. of the IEEE Intl. Conf. on Image Processing (ICIP 2003) (Barcelona, Spain, Sept, 2003).
[2] Gurrin, C., Smeaton, A., Lee, H., Mc Donald, K., Murphy, N., O'Connor, N. and Marlow, S. Mobile Access to the Físchlár-News Archive. In Mobile HCI 2003, Workshop on Mobile and Ubiquitous Information Access (Udine, Italy, Sep 2003).

[3] Harada, S., Naaman, M., Jiun Song M., Wang, Q. and Paepcke, A. Lost in memories: interacting with large photo collections on PDAs. In Proc. of $4^{\text {th }}$ Joint Conf. on Digital Libraries, (JCDL 04) (Tucson, AZ, June, 2004).

[4] Kristoffersen, S. and Ljungberg, F. "Making place" to make IT work: empirical explorations of $\mathrm{HCI}$ for mobile CSCW. In Proc of ACM SIGGROUP Conference on Supporting Group Work (Phoenix, AZ, Nov, 1999).

[5] Longoria, R. Designing mobile applications: challenges, methodologies, and lessons learned. In Proc. of $9^{\text {th }}$ Intl. Conf. on HCI (New Orleans, LA, Aug, 2001).

[6] Marcus, A., Ferrante, J., Kinnunen, T., Kuutti, K. and Sparre, E. Baby faces: user-interface design for small displays. In Proc. of the ACM SIGCHI Conf. on Human Factors in Computing Systems "Making the Impossible Possible", (Los Angeles, CA, April, 1998).

[7] Naaman, M., Harada, S., Wang, Q., Garcia-Molina, H. and Paepcke, A. Context data in geo-reference digital photo collections. In Proc. of $12^{\text {th }}$ ACM Conf. on Multimedia (MM'04) (New York, NY, Oct., 2004).

[8] Pascoe, J., Ryan, N. and Morse, D. Using while moving: HCI issues in fieldwork environments. ACM Trans. on ComputerHuman Interaction (TOCHI), 7, 3, 2000.

[9] Rist, T. A perspective on intelligent information interfaces for mobile users. In Proc. of $9^{\text {th }}$ Intl Conf. on HCI (New Orleans, LA, Aug., 2001).

[10] Rodden, K. and Wood, K. How do people manage their digital photographs? In Proc of SIGCHI Conference on Human Factors in Computing Systems (CHI'03) (Ft. Lauderdale, FL, April, 2003).

[11] Sacher, H. and Loudon, G. Uncovering the new wireless interaction paradigm. ACM interactions, 9, 1, 2002.

[12] Smeulders, A., Worring, M., Santini, S., Gupta, A. and Jain, A. Content-based image retrieval at the end of the early years. IEEE Trans. Pattern Analysis and Machine Intelligence, 22, 12, 1349--1380, 2000.

[13] Thomas, P., Meech, J. and Macredie, R. A Framework for the development of information appliances. In Proc of $20^{\text {th }}$ Annual ACM Symposium on Applied Computing (SAC 2005) (Santa Fe, New Mexico, March, 1995).

[14] Toyama, K., Logan, R., Roseway, A. and Anandan, P. Geographic location tags on digital images. In Proc of $11^{\text {th }}$ ACM Conf. on Multimedia (MM'03) (Berkeley, CA, Nov, 2003).

[15] Wenyin, L., Sun, Y. and Zhang, H. MiAlbum - a system for home photo management using the semi-automatic image annotation approach. In Proc. of $8^{\text {th }}$ ACM Conf. on Multimedia (MM'00) (Marina del Rey, CA, Nov, 2000). 